\title{
La crisis migratoria, la extensión del miedo a través de las TIC e implicación de la Universidad
}

Enviado: 21 de diciembre de 2019 / Aceptado: 10 de enero de 2020

Publicado: 12 de julio de 2020

ELBA ROSA GÓMEZ BARAJAS

Departamento de Ciencias Económicas y Administrativas. Centro Universitario de los Valles, Universidad de Guadalajara, México.

elba.gomez@academicos.udg.mx

\section{RESUMEN}

La presente investigación parte de la crisis que el movimiento migratorio está produciendo en la actualidad, y cómo la sensación de miedo, de libertad y seguridad, fomentado por los medios de comunicación masivos y especialmente por las redes sociales, puede incidir en aumentar la, ya difícil, tarea de integración de los inmigrantes en el país de recepción. La migración es una problemática a escala global, que va a ir en aumento y generando una mayor tensión, por la migración debida al cambio climático y la posible disminución de empleo (a nivel mundial) por el aumento de la robotización del trabajo.

El objetivo es reflexionar (a partir de las aportaciones de organismos internacionales y de autores relevantes) y promover la inclusión de la problemática de la inmigración, en la Universidad, como fenómeno biopsicosocial y geopolítico, relevante en la sociedad actual.

A partir de estas reflexiones y análisis crítico sobre la migración, se exponen algunas de las acciones que

\section{ABSTRACT}

The migration crisis, the extension of fear through ICTs and involvement of the University

The present investigation is based on the crisis that the migratory movement is currently producing, and how the feeling of fear, freedom and security, fostered by the mass media and especially by social networks, can have an impact on increasing difficult, task of integration of immigrants in the receiving country. Migration is a problem on a global scale, which is going to increase and generate greater tension, due to migration due to climate change and the possible decrease in employment (worldwide) due to the increase in work robotization.

The objective is to reflect (based on the contributions of international organizations and relevant authors) and promote the inclusion of immigration issues, at the University, as a biopsychosocial and geopolitical phenomenon, relevant in today's society. 
deberían promoverse a través de la Universidad para la integración de los inmigrantes y otras propuestas de sensibilización al interior de la Instituciones de Educación Superior, sobre cómo abordar este fenómeno de movilización masiva globalizada, como una corresponsabilidad de los agentes que conforman a la Universidad de Guadalajara, creando o retomando iniciativas de las agendas públicas internacionales, más allá de la retórica discursiva.

Palabras Clave: Inmigración, TIC y Universidad.
Based on these reflections and critical analysis on migration, some of the actions that should be promoted through the University for the integration of immigrants and other sensitization proposals within the Higher Education Institutions, on how to address this phenomenon of globalized mass mobilization, as a co-responsibility of the agents that make up the University of Guadalajara, creating or retaking initiatives from international public agendas, beyond discursive rhetoric.

Keywords: Inmigration, ICT, University.

\section{Introducción}

Once años después del inicio de la crisis económica de 2008, originada en Estados Unidos y que tuvo repercusiones en América Latina y en Europa, aún persisten muchos de los efectos que generó. Incluso algunos países, que han entrado en la senda del crecimiento no reflejan un incremento relevante, en los índices de empleo para sus habitantes, lo que ralentiza la recuperación en las esferas sociales, políticas y culturales. Ello arroja sombras importantes para los países, e incuba una colectiva propagación de incertidumbre en las personas en todo el planeta.

Esta crisis social mundial constata la interdependencia a la que están expuestos, en la realidad física y virtual del siglo XXI, todos los países del mundo. La globalización se caracterizó por una mayor libertad para el tránsito de mercancías y de capital, y la fluidez del intercambio (en muchos países) de información a través de las Tecnologías de la Información y de la Comunicación (TIC), especialmente a través de Internet y de los medios de comunicación masiva. Sin embargo, esa libertad de movimientos de capital, mercancía e información no se extendió a los ciudadanos del mundo, cuya movilidad sigue bajo los criterios nacionales e internacionales previos a la globalización.

La globalización, dio lugar a un desarrollo generalizado, aunque no equilibrado, con una afluencia de capitales que ha propiciado el desarrollo de algunos países emergentes. Pero a la vez la deslocalización de empresas, de los países desarrollados, que migran a otros países, buscando trabajadores con salarios más bajos, políticas medio ambientales menos estrictas, y estrategias que permiten gravar menos impuestos o evadirlos incluso. Igualmente, el tránsito de mercancías ha producido un abaratamiento de muchos bienes y productos y un intercambio comercial desregulado, sin precedentes en la historia de la Humanidad. 
La globalización económica y la influencia de las TIC, junto a otros problemas y situaciones como el calentamiento global, el terrorismo, la delincuencia internacional, entre otros, están cambiando fuertemente la concepción de los ciudadanos sobre el mundo, y en algunos aspectos sirviendo como fuente de desasosiego y de pánico colectivo. Dado que es imposible, en un artículo, abordar todas estas problemáticas, vamos a incluir algunas de las más relevantes, según nuestra visión: la migración y el uso de las TIC como generadoras de conflictos.

\subsection{Crisis migratoria}

Las migraciones internacionales hacen aflorar algunas de las consecuencias de la globalización (desequilibrio entre países), y debido a la presencia de las TIC y su consecuente magnificación de la información, las situaciones de otras ciudades y países del mundo son expuestas, de forma inmediata, en las redes sociales y en los medios de comunicación masiva. Por ejemplo, su nivel de vida, su situación laboral, su clima, su infraestructura, el tipo de oportunidades educativas, entre otros, y actúan como anzuelo y reclamo (aunque no sea intencionado) para que ciudadanos de países en situaciones de precariedad sociocultural y económica, más pobres y deprimidos, anhelen migrar a esos países, con la esperanza de mejorar su calidad de vida y la de su familia.

El movimiento migratorio, actual, tiene orígenes y consecuencias distintas, a las de épocas donde no existía la globalización y el impacto de las TIC, tanto por el volumen de personas que se mueven, como las causas y la rapidez de los ciclos económicos. Por ejemplo, países como Ecuador, que tenía un fuerte movimiento migratorio hacia el exterior, en la década de los 90 y en la primera década del siglo XX, lleva ya unos años recibiendo un elevado número de migrantes. Al revés de España, que recibió varios millones de inmigrantes, en las mismas décadas, y hoy parte de su población está migrando.

Son constantes los movimientos de seres humanos de un país a otro, de un continente a otro, en busca de comida, oportunidades de trabajo, mejores servicios y calidad de vida. A ellos se unen así la población que busca paz, huye del terrorismo, del fanatismo religioso y político, del narcotráfico, de las dictaduras en aparentes "sistemas democráticos", de la inequidad, de la impunidad de la delincuencia, de los asesinatos en masa por tecnología bélica, etc. Todos estos sucesos trazan un sendero de desigualdad y miedo que ahora es conocido, casi en tiempo real, debido a las redes sociales, los medios de comunicación y al rol cada vez más activo de una sociedad en hartazgo.

La inmigración, en grandes proporciones, provoca un intercambio de información e influencia entre culturas y con frecuencia un fuerte choque social, ante la presencia de oleadas humanas en territorios ajenos al suyo. "Invasiones" provenientes de otra región, país o continente, provocando la desconfianza en los habitantes de la zona receptora, la inseguridad y el miedo, el crecimiento del racismo, la intole- 
rancia y, en ocasiones, grandes desequilibrios, tanto del país emisor (que pierde con frecuencia parte de la población más joven, con iniciativa y mejor formada) como del país receptor. Para Millán (2016) la migración produce altos niveles de estrés, y los cambios que se propician en los países receptores modifican la demografía, el mercado y puede afectar a la salud mental de sociedades, con las nuevas situaciones y cambios que se producen y los debates que se generan, como los de la identidad colectiva o nacional.

El fuerte movimiento migratorio, especialmente hacia Europa (a pesar de la crisis económica y política que tiene) USA y algunos países asiáticos, genera una gran controversia y dilemas morales, que van a condicionar parte de la agenda mundial y cuya resolución afectará a la Humanidad.

A pesar de la magnitud de los movimientos migratorios, actuales, la tendencia es que va a seguir habiendo un fuerte crecimiento, por los mismos motivos actuales. Pero, además, se van a ir fortaleciendo por el desequilibrio de natalidad, entre los países receptores y los países emisores, y también por las consecuencias del cambio climático, que va a dificultar la vida humana, en distintas zonas del planeta.

Según, Milanovic (2017) que trabaja para el Banco Mundial en temas de desigualdad, el 20 por ciento de la población mundial desea vivir en otro país. Es evidente, que no todos van a movilizarse para cumplir su deseo, pero sí es muy relevante que casi 1.500 millones de personas quieran migrar.

Además, si tenemos en cuenta, que incluso en países con problemas de empleo y que generan un alto movimiento migratorio (México, Marruecos, Turquía...), la automatización o robotización del trabajo va a generar una disminución del empleo, que se va a concentrar en la población joven; y por otro lado, en los países desarrollados también está prevista una disminución del empleo, por esta misma causa (Manyika et al., 2017) es muy fácil que el movimiento migratorio se convierta en un problema social global y central, en un futuro muy cercano.

"El letargo dogmático de las teorías y de los cuerpos teóricos, normativos y descriptivos, no alcanzarán la fugacidad del poder globalizado, por lo que se debe crear un nicho (teórico-empírico) como una categoría ontológica esencial para los estudios de migración" (Estévez, 2018, p. 15).

La anterior afirmación puede ser aplicada a todos los ámbitos, en cuanto a resolución de problemáticas se refiere, y se relaciona directamente con las situaciones adversas a las que se enfrentan, constantemente, los procesos de innovación social. 


\subsubsection{Expansión del miedo al extraño, la inequidad y la violencia}

El terrorismo y la delincuencia internacional están generando una tensión entre libertad y seguridad. Bauman (2015, p. 133) critica cómo el miedo se está cultivando, a través de distintas estrategias de marketing porque "permite recoger abundantes cosechas políticas y comerciales, y el atractivo de una cosecha opulenta inspira a los buscadores de beneficios políticos y comerciales a forzar la apertura de nuevas plantaciones para el cultivo del miedo". Bauman afirma que ese temor hace que un número de personas, cada vez mayor, esté dispuesto a entregar parte de su libertad, para mejorar la sensación de seguridad. Este miedo y estas reacciones se están evidenciando, cada vez más, en el surgimiento de dirigentes políticos y movimientos sociales que fomentan la xenofobia y el rechazo, a las personas que son distintas.

El equilibrio entre libertad y seguridad es expresado por Bauman de la siguiente forma:

La libertad para actuar según los propios deseos, inclinaciones, impulsos y anhelos, y las restricciones impuestas a todo ello en nombre de la seguridad son necesarios para una vida satisfactoria — soportable y habitable - , ya que la seguridad sin libertad equivaldría a la esclavitud, y la libertad sin seguridad significaría el caos, la desorientación, la incertidumbre perpetua, y, en última instancia, la impotencia para actuar resueltamente $(2015$, p. 126).

Otros temores que caracteriza a algunas de las poblaciones de los países más desarrollados, es que la llegada de inmigrantes pueda producir un abaratamiento de salarios y posible pérdida de empleos para la población originaria, y el temor a un incremento de la delincuencia. Esta clase de sentimientos, por parte de la comunidad receptora, tienen como base ideas, en su mayoría infundadas, de que los sujetos que migran son personas sin formación, que no tienen experiencia laboral, que podrían ser personas violentas, delincuentes, etc., y que en general representan un colectivo non grato, que causará desestabilización en las ciudades que conforman el país destino. Estas emociones y sentimientos generan acciones de intolerancia y marginación en la población receptora,

En este apartado, es pertinente introducir el término, acuñado por Adela Cortina (2017), de aporofobia, que la Real Academia de la Lengua Española (2019, s.p.) define como, "fobia hacia personas pobres o desfavorecidas", debido a que es un término que ha pretendido enfatizar su sentido excluyente, y que los migrantes, viajeros o extranjeros que provienen de países que tienen un nivel de vida superior, no son tratados con el mismo grado de marginación, que los de países económicamente más desfavorecidos. Existe entonces, de forma frecuente, una correlación directa entre el rechazo y la marginación y el estatus económico-social-cultural, educativo y laboral de los migrantes. 
Estos hechos y sentimientos, de los que hemos venido hablando en los últimos párrafos, de una parte de los receptores, en contra de los inmigrantes, vulnerándolos y violentando sus derechos humanos más básicos, denotan tanto una falta de información, como de formación de competencias en valores, entre ellas: la solidaridad, ponerse en el lugar del otro, responsabilidad ciudadana, adaptación a situaciones adversas o desconocidas, adaptación al cambio, consciencia universal.

La violencia comunicacional a través del lenguaje es, en nuestros días, un objeto de estudio imprescindible de estudiar y se relaciona con fenómenos de intolerancia y exclusión social, surgidos como respuesta a problemas generados por la globalización. Las TIC potencian la fuerza que ejerce la violencia. Una violencia que no es necesariamente visible y que no es física, ahí radica la perversidad que el poder neoliberal torna en una aparente libertad, de no obediencia y dominio de la vida propia, cuando en realidad subyace un entramado de violencia sutil y no exhibida, para que hagamos de nosotros mismos el binomio víctima-victimario (Han, 2017).

Hay un exceso tanto de información que no informa, como de comunicación basura que no comunica nada. Las personas están separadas por el espacio geográfico, y se acercan a través de las tecnologías y múltiples dispositivos móviles, pero no siempre se genera una información veraz y una comunicación socialmente enriquecedora. Ello provoca la desinformación, justamente en la era informatizada. Además, las personas pueden estar en comunicación y teniendo también la ventaja de no estar cerca, porque conexión no es lo mismo que proximidad (Bauman, 2018; Han, 2017).

El ser humano, como objeto de estudio desde cualquier perspectiva disciplinar, es un ente complejo e impredecible y en constante transformación, por causas internas y externas. Profundizando en el tema del miedo individual y colectivo en la era globalizada, Barrera (2010), hace un recuento histórico de los paradigmas de Darwin, Descartes, Freud y Compte, para concluir con la afirmación de la actual concepción del hombre como un ser biopsicosocial. A través de la integración de mente y cuerpo, y relacionándolo con la interpretación del miedo, desde diversos enfoques, como el biológico, psicológico y sociocultural. Viene a colación en este ensayo su énfasis en la relación de la sociedad y la tecnología, en el caso particular del miedo colectivo, afirma, "la sociedad colabora con el miedo, al magnificar la información alarmista de los medios de comunicación” (Barrera, 2010, p. 5).

Y si lo llevamos al plano de la sociedad globalizada e intercomunicada por las Tecnologías de la Información y de la Comunicación, el mismo autor, nos presenta el panorama complejo del actual en la siguiente aseveración:

Para fines pedagógicos se puede observar que el miedo, el pánico y el miedo colectivo son variaciones de temor, en diferentes niveles de análisis (individual, grupal, intergrupal o colectivo), y es una emoción 
intensa compartida por un grupo o sociedad ante la percepción de un estímulo amenazante, cuyas fuentes pueden ser: psicosociales, políticas, económicas, culturales, espirituales, del cuidado de la salud, de manipulación de los medios de comunicación o más (Barrera, 2010, p. 6).

Según Bauman (2011) todos estos miedos y el malestar social, en todos los aspectos, se está vinculando con los inmigrantes, en todos los países. Para ello, hay que despojar al Otro de su rostro, y una vez reducido a algo, o a un número "su debilidad invita a la violencia con naturalidad y sin esfuerzo" (p. 84).

\title{
1.2. Las TIC: como herramientas de poder en la era del Dataísmo e hipervigilancia informática
}

Para Castells, retomado por Cabero et al. (2016, p. 18) nos encontramos en la Tercera Revolución, a la cual denomina "Informacional", una sociedad organizada de forma peculiar donde la generación, el procesamiento y la transmisión de la información, potenciados por la tecnología se convierten en grandes fuentes de productividad y poder.

En ese mismo sentido, y enfocándose en las diferencias tecnológicas entre países, Cabero et al. (2016, pp. 21-22) enfatizan:

\begin{abstract}
En este nuevo entramado social, la infraestructura tecnológica será la máxima significación; de manera que los países se diferenciarán por el nivel de tecnología que son capaces de diseñar, producir y consumir. De ahí el surgimiento de nuevos conceptos de separación entre los pueblos: inforricos e infopobres; es decir, entre países perfectamente equipados, diseñadores y utilizadores de nuevas tecnologías de la información y comunicación, y países meramente usuarios de tecnologías tradicionales de la información y comunicación e infraequipados.
\end{abstract}

Este tipo de marginación es digital, pero hay, en las dos afirmaciones anteriores, una evidente e irrefutable realidad, la información es poder. El soporte tecnológico, tanto de los países como de los sujetos, se convierte en una herramienta que es utilizada, de manera consciente o inconsciente por los gobiernos, los empresarios, los profesores y directivos de una Universidad, los habitantes de alguna población con intencionalidades positivas o negativas.

La convergencia de las TIC, la globalización y la hiperconexión, genera problemáticas sociales, paradojas culturales, desigualdades económicas, información descontrolada y manipulación. Nos enfrentamos a una ciberviolencia digital, en la que las plataformas sociales están siendo usadas para la 
siembra y cosecha de sentimientos de odio y resentimiento, evidenciándose en acciones negativas como el ciberacoso, ciberbullying, ciberataque, cibersecuestro, ciberextorsión, por parte de la sociedad o del crimen organizado.

Dentro de los TIC, no queremos dejar de resaltar, el alto potencial de las redes sociales y el elevado número de personas que están integradas, en ellas. Solo por resaltar la plataforma social Facebook, hoy en día, tiene más de 2.170 millones de usuarios en el mundo y México, con 85 millones, se posiciona como el quinto país con más seguidores a nivel mundial.

Las redes sociales, y su enorme potencial para comunicar y expandir la información, fueron clave en los movimientos sociales que produjeron el surgimiento de los movimientos de "indignados", en Europa. Que se movilizaron contra la situación económica y denunciaban una falta de democracia real, porque los ciudadanos no estaban empoderados y el poder lo gestionaban las fuerzas económicas y partidos políticos burocratizados, y al servicio de esos poderes económicos. En los países árabes, en la denominada primavera árabe, se evidenció el gran poder de las redes sociales para movilizar a la población (especialmente a los jóvenes) contra gobiernos dictatoriales y corruptos.

En una época de presencia masiva de las TIC, se puede influir en las decisiones de un habitante usuario-digital común, con estrategias para persuadir o disuadir. En épocas electorales para elegir a un mandatario, en la inclinación por un destino de viaje, en la opción de formar o no parte de una comunidad o espacio común, como el caso del Reino Unido y su referéndum, con el caso particular del Brexit, o en decisiones políticas de los ciudadanos de Grecia.

Hay una generación de conflictos que comienzan de manera individual o por una situación concreta, y se va diseminando en comunidades digitales, a grupos de personas, sociedades, países y puede eclosionar en un conflicto, que se moviliza multidireccionalmente con la globalización de la Tecnología.

Otra dimensión en las TIC que ha surgido con fuerza es el Dataísmo o big data. Es un término que Byung Chul-Han, introdujo para referirse a la masificación de los datos, los datos a gran escala o macrodatos dentro de la era de las TIC. Tanto la Inteligencia Artificial (IA), como el Internet de las Cosas (IoT), y el consecuente tratamiento, monitoreo y procesamiento y aplicaciones de esos datos a nivel mundial, son de gran utilidad para predecir el comportamiento de los usuarios. La información es el activo más poderoso incluso mayor que el petróleo (Cabero et al. 2016; Han, 2017; Horizont, 2015).

El documental denominado "Nada es privado", de los directores, Amer y Noujaim (2019), y que se centra en la compañía privada de Cambridge Analítica, habla de la perfilación psicológica que se hace de un usuario, en la hipervigilancia de sus data points almacenados y vistos en tiempo real, en sus redes sociales como Facebook o WhatsApp, y que son una herramienta de información que no sólo invade 
a la privacidad, sino que es un insumo que abre la caja de pandora para la especulación informática, la diseminación del miedo y la propagación de las fakenews.

Visto lo anterior, podemos aducir que las personas en lugar de conectarse se dividen, y que las empresas, que participan en este tipo de acciones, pueden llegar a ser sirvientes, útiles y dóciles para fomentar el autoritarismo y las dictaduras.

El control de la población con el Big Data, la falta de confianza y la distorsión que generan el uso sectario de las TIC, el miedo, la inseguridad que genera el cambio rápido en las sociedades, las limitaciones crecientes de los gobiernos para atender las necesidades sociales de sus ciudadanos, junto al fenómeno de la migración introduce a las sociedades en una situación de riesgo, que hay que gestionar adecuadamente, sin perder la vista del Otro. Las Universidades tienen que implicarse en este tema crucial y en ello nos adentraremos en el apartado 3.

\section{Objetivos de la investigación}

Reflexionar y promover la inclusión de la problemática de la inmigración, en la Universidad, como fenómeno social relevante en la sociedad actual globalizada, teniendo en cuenta el alto poder de las TIC, para, a partir de ello, plantear e implementar propuestas, para la integración de los inmigrantes en la sociedad que los recibe, produciéndose un enriquecimiento mutuo.

\section{Discusión y conclusiones}

Con frecuencia la innovación se suele vincular con la innovación tecnológica y las aplicaciones que proceden de la investigación básica en el campo de las Ciencias Naturales e Ingeniería. En contra de esta forma de entender la innovación desde una perspectiva meramente economicista y tecnologicista, exponen su postura más holística, Stiglitz (premio nobel de economía) y Greenwald (uno de los inversores más reconocidos en Wall Street):

Las innovaciones sociales no son menos importantes que las tecnológicas en las cuales se enfocan tradicionalmente los economistas. El progreso de la sociedad humana tiene tanto que ver con dichas innovaciones -incluidas las relacionadas con cómo administrar grandes organizaciones y el aprendizaje organizacional y cómo promover el aprendizaje social de manera más amplia, incluso mediante las políticas industriales y comerciales - como son las mejoras en la tecnología (Stiglitz y Greenwald, 2015, p. 501). 
La Universidad ha sido y es el seno social de la producción del conocimiento, el cual es definido por la Fundación Europea de la Ciencia, de esta manera:

El conocimiento es el núcleo de todo debate sobre el aprendizaje y cabe entenderlo como el modo en que los individuos y las sociedades dan un sentido a la experiencia, por lo que se puede considerar en términos generales como la información, el entendimiento, las competencias, los valores y las actitudes adquiridos mediante el aprendizaje. El conocimiento como tal está indisolublemente ligado a los contextos culturales, sociales, ambientales e institucionales en los que se crea y reproduce (UNESCO, 2015a, p. 18).

El conocimiento generado en la universidad es un insumo para la economía y la sociedad. Por tanto, una sociedad cuya economía se cimenta en el conocimiento, que es generado por ideas y desarrollos del aprendizaje y del capital intelectual, y además complementada con el uso de las TIC como herramienta, es capaz de conseguir verdaderas innovaciones, logrando cambios estructurales en la relación que siempre guarda la complejidad del sistema económico y político-laboral con la educación (Valenti, 2008).

La Universidad debe adaptarse a una sociedad en cambio permanente, pero no ser arrastrada por el mismo. Es decir, debe ser siempre un ente analítico, reflexivo, crítico, y propositivo siendo un agente fundamental, tanto del cambio como de la direccionalidad de este. Por ello, estimamos que la Universidad es clave para abordar la crisis migratoria y el uso adecuado de las TIC.

\subsection{Las crisis como nichos de oportunidad para las Innovaciones, de cara al replan- teamiento de la sociedad, a través de la Universidad}

Es a partir de las crisis que, desde otras etapas históricas, se han modificado las acciones humanas para una mejora del bien común.

En la crisis migratoria, tiene cabida un intercambio multicultural y multirracial. El migrante durante su travesía, experimenta una gran variedad de sentimientos y experiencias, que no elige vivir: depresión, Síndrome de Ulises (trastorno de inmigrante caracterizado por estrés crónico y múltiple), añoranza de la familia y de la tierra de origen, angustia por la desintegración familiar, hambre y frío, rechazo, estigma, marginación y vulnerabilidad. En este fenómeno, la Universidad debe tener una solidaridad real con los

migrantes. De frente a la actual crisis migratoria, a nivel mundial, deben implementarse estrategias de sensibilización y de concientización, tanto para la comunidad receptora como para los individuos que llegan a ella. ¿Qué responsabilidad tienen la Universidad en este fenómeno? ¿De qué manera abordaría el fenómeno migratorio? ¿A través de sus colectivos de investigadores, docentes, alumnos y directivos? ¿Es a través de la Formación Integral y mediante talleres y cursos de formación sobre migración, 
inclusión, tolerancia, respeto a la diversidad y temáticas que no están disociadas? ¿Es acaso que con la introducción de una LGAC dentro de sus Cuerpos Académicos se puede incursionar en el estudio de los contextos específicos, desde donde se pueden abordar los impactos negativos y los beneficios que tiene la inminente crisis migratoria mundial?

En el caso del estado de Jalisco, México. ¿Cómo debería abordar la Universidad de Guadalajara los problemas de un México emigrante y un México como país de tránsito o país destino? A continuación, se especifican algunas propuestas, que podrían servir para implicar, a la Universidad de Guadalajara y a otras universidades, en un tema de tanta relevancia geopolítica y biopsicosocial:

- Formación en competencias universales, para alumnos, profesores, directivos, a través de Talleres comunitarios en los municipios donde la Universidad tiene injerencia, para sensibilizar a jóvenes, mujeres, niños y adultos mayores.

- Divulgación de las competencias en valores: Apreciación de la diversidad social y cultural, preservación y valoración de la identidad cultural de origen en el marco de la cultura universal; compromiso ético y solidario; compromiso social; capacidad de servir al bienestar social y ambiental, así como a la resolución de los problemas de interés nacional; actuar con servicio de ciudadanía, vocación de servicio público y equidad; capacidad de ser actor del cambio participando en forma democrática y responsable en los procesos de transformación del país; valoración del medio ambiente y la biodiversidad; sensibilidad para comprender y actuar ante problemas universales: medio ambiente, conflictos y desastres y actitud humanista (Beneitone et al., 2007).

- Involucramiento con empresas, hospitales, ONG's, con los que la Universidad tenga convenio y relación, para actuar de forma coordinada ante el fenómeno migratorio.

- Posibilitar la realización del servicio social, de prácticas profesionales, etc., de alumnos de pregrado en actividades relacionadas con la migración.

Desde una óptica más tolerante y de cohesión social, promovida por la ONU (2017) a través de la NAU (Nueva Agenda Urbana), partiendo de los ODS (Objetivos del Desarrollo Sostenible), y de la Educación para el 2030, se plantea la crisis migratoria como un fenómeno que es corresponsabilidad de todos los países del mundo, de las Universidades y de las instancias gubernamentales o corporativos empresariales. Ello, abre una gama de posibilidades de innovación mundial, pues reta al replanteamiento de los discursos y de la retórica, para encarar las multifactoriales problemáticas actuales, que se convierten en causa de la migración masiva actual, y dar paso a la acción, a la investigación, a incluir esas temáticas dentro del currículo de todos los niveles educativos, o dentro de la formación integral o continua y de la educación para toda la vida. Es imperativo hacer una reingeniería de la no incluyente "aldea global". 
Las regulaciones que están surgiendo, ante el fenómeno migratorio, a nivel mundial, son algunas de las ventajas derivadas de esta movilización masiva sin precedente, entre un mundo que se autodenomina globalizado y de "libertad de apertura". Pero que debido a la intolerancia sociocultural y el abuso de las autoridades o de los mismos individuos, ahora se está replanteando normar el flujo migratorio en pro de la seguridad de los migrantes. De acuerdo, al "Pacto Mundial para la Migración Segura, Ordenada y Regular" promovido por la ONU (2018), existen regulaciones garantistas para los migrantes y dentro de los 23 objetivos que se ha planteado, destacamos los siguientes: medidas contra la trata y el tráfico de personas, evitar la separación de las familias, usar la detención de migrantes sólo como última opción, reconocimiento al derecho de los migrantes irregulares a recibir salud y educación en sus países de destino.

Enlistemos ahora otros de los beneficios del movimiento migratorio. Los inmigrantes también contribuyen con el PIB del país destino, como favorecen a la dinamización económica y social de las ciudades de tránsito o ciudades santuario, si hablamos en términos de micro indicadores. En esta línea Tirole (2017) premio nobel de Economía, reflexionando sobre la crisis y debates que hay en la Unión Europea por el fenómeno migratorio, recoge el miedo que genera los migrantes por la escasez de empleo, y considera que si no se controla el calentamiento global aumentará la migración. No obstante, Tirole cree que deben reformarse las instituciones europeas para acogerlos con dignidad y no excluirlos del mercado laboral, valorando su aportación al crecimiento económico de la Unión Europea y su aportación cultural.

Para Stiglitz y Greenwald (2015), los movimientos migratorios pueden facilitar la movilidad del conocimiento y aprendizaje, entre los países, ayudando a crear una economía del aprendizaje.

El intercambio sociocultural, ideológico, la cosmovisión del país de origen y la identidad del inmigrante, entra en contacto con la identidad cultural de los residentes locales y debe generar una identidad colectiva de mayor riqueza, fundada en la diversidad, precepto que, a decir por la UNESCO (2005), es uno de los pilares del desarrollo incluyente, megadiverso e integral de todos los pueblos del mundo.

La Declaración de Incheon, con su prospectiva de Educación 2030, habla de la educación incluyente, "colaborar para que todos los niños tengan acceso a oportunidades de aprendizaje y educación de calidad durante su vida, independientemente del lugar donde hayan nacido, de su sexo o de los ingresos familiares" (UNESCO, 2015b, p. 12). Y, además, de otros derechos humanos básicos como, igualdad de género, de derecho a la paz, prosperidad compartida, inculcar valores de justicia y paz y de derechos humanos y propone metas, parece ser la némesis de la realidad actual.

Es probable que la globalización, de los problemas, nos lleve a crear nuevas organizaciones más flexibles e iniciar un camino hacia una gobernanza mundial. Ello no será posible, si además de la conexión que han propiciado las TIC no se generan sentimientos de empatía entre las personas, pueblos y naciones, que conforman la Humanidad. La Universidad es un espacio adecuado para crear estas 
corrientes, y, en parte, ya lo realiza a través de los intercambios de estudiantes y profesores. Pero, debe ir más allá y contemplar este aspecto en los contenidos, puesto que muchos alumnos no participarán en intercambios.

Como hemos señalado, anteriormente, las TIC se han utilizado como herramientas para transmitir miedo y generar odio, y, por otro lado, son ventanas abiertas que animan a ciudadanos castigados por la pobreza e inseguridad a dejar sus tierras y familias. Las TIC han permitido, a regiones y países, entrar en una nueva era de comunicación, y han abierto la posibilidad de que el mundo sea efectivamente una aldea global. Sin embargo, uno de los desafíos más importantes de la globalización es la libre circulación de personas.

Ante situaciones de inequidad, de malas prácticas con TIC, violencia dentro de las Instituciones Educativas de Educación Superior, violencia y discriminación social, la Universidad debe solidarizarse, no solamente en discursos mediáticos, sino enfrentando, de forma pronta y eficaz, las situaciones en donde pueda o deba intervenir. Utilizando su capital humano pensante, investigadores, docentes, directivos, administrativos, alumnos y a través de los convenios con hospitales, empresas, y ONG’s, para establecer mecanismos de ayuda a grupos vulnerados, a problemáticas sociales y desastres naturales, epidemias, etc.

Estas nuevas posibilidades, problemáticas y necesidades formativas, han abierto y abrirán nuevos campos de actuación para las Universidades y para la innovación educativa, entre ellos los siguientes:

- La divulgación y diseminación del conocimiento a la sociedad. Las Universidades deben asumir la misión de transitar hacia un conocimiento divulgativo, asequible para la sociedad.

- La formación permanente de egresados, a través de enseñanzas especializadas. Las TIC aumentan las posibilidades de esta formación especializada que sea más incluyente. La mayoría de las Universidades han adoptado plataformas virtuales, que hacen posible que se puedan impartir formación totalmente a distancia, o bien semipresencial. Favoreciendo que personas con dificultades de tiempo o para desplazase puedan recibir una formación de calidad y especializada.

- Incluir contenidos relacionados con las competencias genéricas, educación para la vida, inteligencia emocional, resiliencia, educación ciudadana, etc., sin perder de vista la formación disciplinar.

Si la sociedad se encuentra en tiempos revueltos y turbulencias político-económicas, la Universidad, como cuna de la educación, debe adaptarse a esos cambios. De forma que contribuya a la solución de problemáticas específicas, desde las más inmediatas hasta las de mediano plazo, revistiendo a su profesorado y su alumnado con una malla intelectual capaz de hacer frente a las situaciones que encuentren a su paso. 
La educación, en la actualidad, debe atender a las necesidades no solo cognitivas de sus estudiantes, sino además debe promover, en ellos, una educación que los forme como seres humanistas. Los provea de habilidades sociales y culturales, cada vez más universales, sin perder de vista la identidad local, los valores endógenos y los nuevos retos de la educación del siglo XXI.

\section{Referencias bibliográficas}

Amer, K. Kos, P. Dreyfous, G. Korin, J. (productores) y Amer, K. y Noujaim, J. (directores). (2019). "The Great Hack". "Nada es Privado". (Documental). EU.: Netflix. Recuperado de https:/www.netflix.com/watch/80117542?trackId=13752289\&tctx $=0 \% 2 \mathrm{C} 0 \% 2 \mathrm{Cf} 469 \mathrm{f}$ 5286a912a50974144f905c0a077d757d8cc\%3A66f2eeffa5b4c0717cd7738b7570ce13f $\underline{5 \mathrm{~d} 8 \mathrm{~d} 441 \% 2 \mathrm{C} \% 2 \mathrm{C}}$

Barrera, J.A. (2010). El miedo colectivo: el paso de la experiencia individual a la experiencia colectiva. Recuperado de http://www.elcotidianoenlinea.com.mx/pdf/15902.pdf

Bauman,Z. (2011). Daños colaterales. Desigualdades sociales en la era global. México: Fondo de Cultura Económica.

Bauman, Z. (2015). Entre el miedo y la indiferencia: la pérdida de sensibilidad. En Bauman, Z. y Donskis, L. Ceguera moral. La pérdida de sensibilidad en la modernidad líquida (pp. 121-164). Barcelona: Paidós.

Bauman, Z. y Leoncini, T. (2018). Generación líquida. Transformaciones en la era 3.0. Barcelona: Paidós.

Beneitone, P. et al. (2007). Reflexiones y perspectivas de la Educación Superior en América Latina. Informe final Proyecto Tuning América Latina 2004-2007. Bilbao: Universidad de Deusto y Universidad de Groningen. Recuperado de http://www.google.com.mx/search $? \mathrm{hl}=\mathrm{es} \&$ source $=\mathrm{hp} \& \mathrm{q}=\mathrm{la}+$ nocion + por + competencia $+\mathrm{en}+\mathrm{el}+$ proyecto+tuning + menend $\underline{\mathrm{ez}}+2009 \& \mathrm{aq}=\mathrm{f} \& \mathrm{aqi}=\& \mathrm{aql}=\&$ oq $=\& \mathrm{gs}$ rfai

Cabero, J.; Leiva, J.J.; Moreno, N. et Al. (2016). Realidad aumentada y educación: Innovación en contextos formativos. Barcelona: Octaedro.

Cortina, A. (2017). Aporofobia, el rechazo al pobre. Un desafío para la democracia. Barcelona: Paidós. 
Estévez, A. (2018). Guerras necropolíticas y biopolíticas de asilo en América del Norte. Universidad Nacional Autónoma de México. México: UNAM.

Han, B .-C. (2017). Topología de la violencia. Barcelona: Herder

Horizon (2015). Informe Horizon. Educación Superior 2015. Recuperado de http://cdn.nmc.org/ media/2015-nmc-horizon-report-HE-EN.pdf

Manyika, J. (2017). Jobs Lost, Jobs Gained: Workforce transitions in a time of automation. McKinsey Global Institute.

Milanovic, B. (2017). Desigualdad mundial. Un nuevo enfoque para la era de globalización. México: Fondo de Cultura Económica.

Millán, J. A. (2016). La globalización de las conductas: una reflexión prospectiva. En Miklos, T. y Arroyo M. (coords.). El futuro a debate. Respuestas prospectivas y estratégicas ante la incertidumbre global (pp. 229-261). México: Limusa y Gobierno del Estado de México.

ONU (2017). Nueva Agenda Urbana. Recuperado de http://habitat3.org/wp-content/uploads/NUASpanish.pdf

ONU (2018). Pacto Mundial para la Migración Segura Ordenada y Regular. Recuperado de https:// www.refworld.org.es/pdfid/5c0eac944.pdf

Real Academia de la Lengua Española (2019). Diccionario. Recuperado de https://dle.rae. $\underline{\text { es} / ? \mathrm{id}=3 \mathrm{FfFecJ}}$

Stiglitz, J. E. \& Greenwald, B. C. (2015). La creación de una sociedad del aprendizaje. México: Crítica.

Tirole, J. (2017). La economía del bien común. Barcelona: Penguin Randon House Grupo Editorial.

UNESCO (2005). Convención sobre la protección y promoción de la diversidad de las expresiones culturales. Recuperado de http://unesdoc.unesco.org/images/0014/001429/142919s.pdf

UNESCO (2015a). Replantear la Educación ¿Hacia un bien común mundial? Recuperado de http:// www.eduteka.org/pdfdir/unesco-replantear-educacion.pdf

UNESCO (2015b). Declaración de Incheon. Recuperado de http://unesdoc.unesco.org/ images/0023/002338/233813m.pdf

Valenti, G. (2008). Ciencia, tecnología e Innovación. Hacia una agenda de política pública. FLACSO: México. 